\title{
Autonomic dysfunction in women with fibromyalgia
}

\author{
J Derek Kingsley* \\ See related research by Ribeiro et al., http://arthritis-research.com/content/13/6/R190
}

\begin{abstract}
Fibromyalgia (FM) is an idiopathic disease characterized by widespread pain and a myriad of symptoms. Symptoms are diverse and include not only pain but also anxiety, depression, orthostatic intolerance, and cold intolerance. While the etiology of FM is not fully understood, data have suggested that FM may stem from dysfunction of the autonomic nervous system. This dysfunction has been reported at rest, and after a physiological stressor such as exercise. However, few studies have examined the responses during exercise. This novel approach may shed some new light on the effect of exercise in women with FM.
\end{abstract}

In a recent issue of Arthritis Research \& Therapy, Ribeiro and colleagues [1] present data that lend further support to the theory that women with fibromyalgia (FM) suffer from autonomic dysfunction in response to, and during recovery from, acute exercise. Alterations in autonomic function have been suggested to increase the risk for cardiovascular events and mortality [2]. While the data are not fully understood regarding the risk of premature cardiovascular disease in women with FM specifically, it appears that there is definitive evidence to support autonomic dysfunction at rest.

The study by Ribeiro and colleagues examined cardiovascular responses during and after a maximal treadmill test in women with FM and healthy controls. Chronotropic incompetence, the inability to increase heart rate with increasing exercise intensities, was used as a measure of cardiac autonomic responses during the exercise bout. Meanwhile, heart rate recovery taken at 1 and 2 minutes post-exercise was used to assess recovery from the acute aerobic exercise bout.

The major findings of this study were relatively novel. The present study reported that women with FM had a

*Correspondence: Derek.Kingsley@indstate.edu

Indiana State University, Arena C-23, Terre Haute, IN 47809, USA lower oxygen consumption (VO2max) than healthy controls, which has been reported previously [3]. More importantly, Ribeiro and colleagues reported that chronotropic reserve was significantly lower in women with FM than healthy controls. Furthermore, 57.1\% of the women with FM presented chronotropic incompetence compared to none of the healthy controls. Lastly, there was a significant reduction in heart rate recovery at 1 minute and 2 minutes after the exercise bout in the women with FM compared to healthy controls, suggesting an inability of the parasympathetic system to recover.

One of the main points highlighted in the paper by Ribeiro and colleagues is the lack of data regarding autonomic modulation during exercise in women with FM. The only study that I am aware of that has attempted to quantify autonomic function during exercise in patients with FM is by Van Denderen and colleagues [4]. These authors noted that patients with FM had lower heart rates and had lower levels of circulating catecholamines at the same absolute workload compared to healthy controls. Other studies have demonstrated altered muscle function during aerobic exercise in women with FM, which may be related to autonomic dysfunction, but it was not measured.

Since the study by Van Denderen and colleagues, other reports have demonstrated that women with FM have autonomic dysfunction at rest [5,6]. In addition, our laboratory has previously suggested that autonomic modulation is altered in women with FM during recovery from an acute bout of resistance exercise [7] as well as during recovery from isometric handgrip [8]. However, Cohen and colleagues [5] and data from our laboratory utilize heart rate variability, fluctuations in the $R-R$ interval on an electrocardiogram, as a method to investigate autonomic dysfunction that has limited use during an exercise bout. However, it appears that autonomic modulation is impaired after exercise, either aerobic exercise or resistance exercise, regardless of the method utilized to investigate it.

Martinez-Lavin and Hermosillo [9] have suggested that autonomic dysfunction may explain many of the symptoms of FM, such as insomnia, irritable bowel syndrome, 
anxiety, orthostatic intolerance and fatigue as well as pain. Furthermore, data suggest that women with FM experience hyperactivity of the sympathetic system at rest, and hypoactivity of the sympathetic nervous system during a stressor, such as exercise, standing or cold exposure. This finding is supported by our laboratory and others [9].

Currently, there are no blood markers for FM. The 'revised' criterion for diagnosis of FM is based on a widespread pain index and a symptom severity scale [10]. The issue with the autonomic testing is that interpretation of the data is non-specific. To further clarify, it has been suggested that body fat alters autonomic function in an otherwise healthy individual. In addition, it has also been suggested that $60 \%$ of all women with FM are overweight/ obese as determined by body mass index [11]. While our laboratory has reported autonomic dysfunction in overweight women (body mass index of 25 to $29.9 \mathrm{~kg} / \mathrm{m}^{2}$ ) with FM [6,7], no one has assessed autonomic function in lean women with FM compared to obese women with FM. The paper by Ribeiro and colleagues also utilized overweight women with FM. However, all of these data may be influenced by the body composition of the participants, independent of FM.

In conclusion, while it appears that autonomic dysfunction is associated with FM at rest, the data regarding responses following an exercise bout are severely limited. In the article by Ribeiro and colleagues, only $57 \%$ of the participants experienced the inability to increase heart rate with exercise. In addition, the participants also had an inability to recover from exercise. Ultimately, the prevalence of cardiovascular disease in women with FM is unknown. However, Ribeiro and colleagues and our laboratory suggest that the responses of the autonomic nervous system to acute exercise, aerobic or strength, may predispose women with FM to a cardiovascular event post-exercise. However, at this point in time the consequence of autonomic dysfunction after exercise on the development of cardiovascular events is not fully understood. Therefore, more research needs to focus on autonomic modulation in women with FM after an acute bout of exercise to elucidate if these responses truly increase the risk for a cardiovascular event.

\section{Abbreviations}

FM, fibromyalgia; VO2max, oxygen consumption.

\section{Competing interests}

The author declares that he has no competing interests.

Published: 20 February 2012

\section{References}

1. da Cunha Ribeiro RP, Roschel H, Artioli GG, Dassouki T, Perandini LA, Calich AL, de Sá Pinto AL, Lima FR, Bonfá E, Gualano B: Cardiac autonomic impairment and chronotropic incompetence in fibromyalgia. Arthritis Res Ther 2011, 13:R190.

2. Tsuji H, Larson MG, Venditti FJ Jr, Manders ES, Evans JC, Feldman CL, Levy D: Impact of reduced heart rate variability on risk for cardiac events. The Framingham Heart Study. Circulation 1996, 94:2850-2855.

3. Lund E, Kendall SA, Janerot-Sjoberg B, Bengtsson A: Muscle metabolism in fibromyalgia studied by $\mathrm{P}-31$ magnetic resonance spectroscopy during aerobic and anaerobic exercise. Scand J Rheumato/ 2003, 32:138-145.

4. Van Denderen JC, Boersma JW, Zeinstra P, Hollander AP, van Neerbos BR: Physiological effects of exhaustive physical exercise in primary fibromyalgia syndrome (PFS) : is PFS a disorder of neuroendocrine reactivity? Scand J Rheumatol 1992, 21:35-37.

5. Cohen H, Neumann L, Kotler M, Buskila D: Autonomic nervous system derangement in fibromyalgia syndrome and related disorders. Israel Med Assoc J 2001, 3:755-760.

6. Figueroa A, Kingsley JD, McMillan V, Panton LB: Resistance exercise training improves heart rate variability in women with fibromyalgia. Clin Physiol Funct Imaging 2008, 28:49-54.

7. Kingsley JD, Panton LB, McMillan V, Figueroa A: Cardiovascular autonomic modulation after acute resistance exercise in women with fibromyalgia. Arch Phys Med Rehabil 2009, 90:1628-1634.

8. Kingsley JD, Panton L, Lee J, McMillan V, Fernhall B, Figueroa A: Exercise pressor response in overweight and obese women with fibromyalgia. Med SciSports Exercise 2006, 38:\$320.

9. Martinez-Lavin M, Hermosillo AG: Autonomic nervous system dysfunction may explain the multisystem features of fibromyalgia. Semin Arthritis Rheum 2000, 29:197-199.

10. Wolfe F, Clauw DJ, Fitzcharles MA, Goldenberg DL, Katz RS, Mease P, Russell AS, Russell IJ, Winfield JB, Yunus MB: The American College of Rheumatology preliminary diagnostic criteria for fibromyalgia and measurement of symptom severity. Arthritis Care Res (Hoboken) 2010, 62:600-610.

11. Yunus MB, Arslan S, Aldag JC: Relationship between body mass index and fibromyalgia features. Scand J Rheumatol 2002, 31:27-31.

doi:10.1186/ar3728

Cite this article as: Kingsley JD: Autonomic dysfunction in women with fibromyalgia. Arthritis Research \& Therapy 2012, 14:103. 